\title{
Design and development of driving simulator scenarios for road validation studies
}

\author{
Juan F. Dols \\ Instituto de Diseño y Fabricación (IDF), Universitat Politècnica de València, España \\ Jaime Molina \\ IDF, Universitat Politècnica de València, España \\ Francisco Javier Camacho-Torregrosa \\ Grupo de Investigación en Ingeniería de Carreteras (GIIC), Universitat Politècnica de \\ València, España \\ Javier Marín-Morales \\ LabHuman, Universitat Politècnica de València, España \\ Ana María Pérez-Zuriaga \\ GIIC, Universitat Politècnica de València, España \\ Alfredo García \\ GIIC, Universitat Politècnica de València, España
}

\begin{abstract}
In recent years, the number of road-based studies using driving simulators is growing significantly. This allows evaluating controlled situations that otherwise would require disproportionate observations in time and/or cost. The Institute of Design and Manufacturing (IDF) of the Polytechnic University of Valencia (UPV) has developed, in collaboration with the Engineering Research Group Highway (GIIC) of the UPV, a low cost simulator that allows rapid implementation and effectively a new methodology for validation studies of different roads through the implementation in the simulator scenarios of existing roads. This methodology allows the development of new scenarios based on the analysis of a layers-file system. Each layer includes different information from the road, such as mapping, geometry, signaling, aerial photos, etc. The creation of the simulated scenario is very fast based on the geometric design software, making it easier to consulting firms using the system that can evaluate and audit a particular route, obtaining reliable conclusions at minimal cost, even if the road is not actually built. This paper describes the basic structure of the layers generated for developing scenarios and guidelines for the implementation thereof. Finally the application of this methodology to a case of success will be described.
\end{abstract}




\section{INTRODUCTION}

Simulation can be described as a method of reproducing a situation similar to reality, yet controllable. To be achieved, it is necessary to reproduce an environment with identical stimuli to a real situation. This feature converts a simulator into a flexible scientific research tool - assisting laboratory experiments that could be expensive, dangerous, or unrepeatable in the real world. In the field of driving, simulations are performed with driving simulators. These devices generate situations requiring the same responses as reallife driving - but without the risks of being on the road.

The basis for assessing any simulator is applicability. Simulators must be designed and equipped according to their intended use. Driving simulators have been developed enormously over the past years. Actually exist versions ranging from PC-based models to high-level models such as the Daimler-Benz (Nordmark, 1990; Weir and Clark, 1995). By using driving simulators, a wide variety of studies can be undertaken regarding: the driver; the vehicle; and the road.

Sometimes it is useful to categorize driving simulator research according to experimental variables instead of study objectives. These variables are in accordance: the driver; the vehicle; the environment; and the road. Examples of these types of studies can be seen in Table 1. Basically, the advantages of using simulators as a research tool can be summarized as: experimental works can be closely defined and easily repeated, parameters and experimental variables can be both easily modified and stored, the effects of driver fatigue can be safely studied, accidental or unpredictable situation can be analyzed safely, and prototypes and series design decisions can be made at an earlier phase of development.

\begin{tabular}{|c|c|c|}
\hline $\begin{array}{c}\text { DRIVER RELATED } \\
\text { STUDIES }\end{array}$ & $\begin{array}{c}\text { VEHICLE RELATED } \\
\text { STUDIES }\end{array}$ & $\begin{array}{c}\text { ROAD \& } \\
\text { ENVIRONMENT }\end{array}$ \\
\hline $\begin{array}{l}\text { Drugs, age, fatigue, effects } \\
\text { of medical treatment, mental } \\
\text { and emotional states, } \\
\text { alertness, personality } \\
\text { aspects, mental and physical } \\
\text { disabilities, other medical } \\
\text { conditions, risk-taking } \\
\text { mentality, training, } \\
\text { warnings, information, etc. }\end{array}$ & $\begin{array}{l}\text { Vehicle type, dynamic } \\
\text { qualities, control response, } \\
\text { information mechanisms } \\
\text { (form, content and } \\
\text { location), field of exterior } \\
\text { vision, restraint systems, } \\
\text { interior environmental } \\
\text { factors, passengers, ADAS, } \\
\text { V2X, etc. }\end{array}$ & $\begin{array}{l}\text { Road geometry, road } \\
\text { markings, surface properties, } \\
\text { road structure elements, } \\
\text { visual texture, color, } \\
\text { indications, vehicle speed, } \\
\text { traffic regulators, lighting, } \\
\text { weather, pedestrian and other } \\
\text { vehicles, obstacles, off-road } \\
\text { handling, etc. }\end{array}$ \\
\hline
\end{tabular}

Table 1. Type of studies undertaken with simulators depending on selected variables.

In accordance with more modern thinking, the driving simulation is regarded as a tool for promoting risk awareness and a way of allowing the driver candidate to try out various 
driving situations which cannot be planned in regular traffic or which would involve excessive danger on the road (Verwey, 1995). However, the purpose of the simulator in many human factors studies is to detect differences in performance produced by changes in the subject's capabilities (e.g. under the influence of alcohol or reduced capabilities due to illness or disability) or differences in secondary-task loading (e.g. use of an in-vehicle route-guidance system) (Read and Green, 1999).

A simulator with a good fidelity should be able to train the basic psychomotor aspects (control of the steering and speed) under complex conditions. Even more important, a simulator with a good control in the scenarios can teach a wide range of cognitive abilities that are required to deal with complex roads and conditions of difficult traffic, including appropriates situation awareness, hazard perception, decision taking and defensive techniques of driving (Allen et al. 2000).

The use of driving simulators in studies of road design, mainly to analyze the influence of geometry in driver behavior has been widely performed by different researchers, both affecting the coordination of the horizontal layout and elevation, cross section analysis, overtaking maneuvers, access to acceleration and deceleration lanes, intersection design and signaling.

In addition, driving simulators constitutes a very useful tool to study road safety taking into account the human factors, as they may be able to generate virtual scenarios where the driver can act similarly as it would do in a real road. The road to analyze may have already been built or being under design, to be evaluated from a safety perspective. In this case, driving simulators can introduce a better analysis due to research developed would allow to obtain data at a lower cost, lesser risk and greater control over the variables under study, mainly speed. The analysis of a virtual road that only exists in the early design phase in a driving simulator, it provides an accurate risk assessment; in this case, the virtual scenario representing the road geometric design, is crucial.

But depending on the objectives pursued in the research, the modeling scenario procedure requires a very careful control of the environmental conditions to reproduce. This design can be considered one of the most critical steps, constituting a real bottleneck in the implementation of experiments (Bhatti et al., 2012). The definition of where and how it will occur a series of events in the simulated environment and the characteristics of objects - road profile and geometry, road signs and markings, geographical environment, textures and colors, lighting, shadows, etc.- will determine the success or failure of the scheduled experiments.

Scenario modeling and specification requires knowledge of both traffic characteristics and conditions of the simulation, to be as close as possible to the real world. In addition, virtual scenarios are being increasingly more complex and sophisticated, to assess for example, 
the use of advanced driver assistance (ADAS), or even the evaluation of vehicleinfrastructure or vehicle-to-vehicle communication (V2X) (Hiblot et al., 2010). Thus, as suggested by Schindler and Hesse (2014), the design process of virtual driving scenarios with a certain degree of complexity, should meet three basic recommendations:

- Each part of the scenario should be created and designed separately, rather than a complete scenario.

- The different parts of the scenario should be developed in rapid iterations, allowing exploration of different parameters and alternatives, generating high-quality scenarios.

- Requested scenario receptors should participate actively in the design process thereof, collaborating with developers who will implement the source code.

Creation of databases with information on the road network to be used for the scenario design, it has traditionally required the use of different tools, not standardized and with different commercial origin. This generates a process of incomplete development with excessive cost-time and no exportable results to other simulation tools. Thus, market driving scenarios developers have been looking for during time the way to implement newer advanced design tools for simulation scenarios that would allow interoperability between databases and experimental simulation tools, both for training and research applications. This in fact means that the use of databases with interchangeable format and basic information could be used by different users and experimental tools.

An example of this trend is RoadXML software, developed by Oktal, whose main objective is to use a single data format for all modules of the simulator. The format used is based on XML files type and is designed to be flexible and extensible so that any user could improve the characteristics of the road network used, even with your own data. It is therefore an open database for road design format, whose structure is composed by different layers of data, each of which represents a type of differentiated information (Chaplier et al., 2010). These layers are divided into 4 levels of information:

- Topology: location of components and connections to the road network (lines distance, relative position to road axis, road width and distance to road axis).

- Logic: elements information in the road environment (road signaling to adjust traffic preferences, direction of movement, vehicles authorization).

- Physical: characteristics of elements (surface material properties of the road and obstacles).

- Visual: 3D elements representation (road markings width, texture, 3D objects representation, elevation profile, etc.).

It is difficult to find out scenarios in RoadXML format. There are libraries open to read data in this format programming, but there is only one software for editing (SCANER studio ${ }^{\mathrm{TM}}$ software released by Oktal). 
Another example of this methodology is the open format OpenDRIVE, developed by VIRES for the multinational Daimler AG. The architecture of this software is also based on the use of different layers of data that are inter-exchanged between several car manufacturer companies in collaboration. Such layers describe the relative position of other vehicles and pedestrians to simulated vehicle, road geometry characteristics, position of traffic signals, etc. (Dupuis et al., 2010).

Both solutions offer the possibility of working with open access software that theoretically enables the exchange of formats from databases for simulation environments design. However, in both cases, the RoadXML format developed by Oktal, and OpenDrive from VIRES, the participation and collaboration of an intermediate agent that manages access to simulation scenario file sharing is needed. This fact could be seen as a barrier and a real drawback for interoperability of file scenario databases between the market agents, users and developers, as always would be needed to filter the developed scenarios throughout private market companies.

Taking into account this new methodology for developing road simulation scenarios, based on the analysis of multiple layers-file format system, the Institute of Design and Manufacturing (IDF) of the Polytechnic University of Valencia (UPV), in collaboration with the Engineering Research Group Highway (GIIC) of the UPV, has created their own scenarios design procedure that have been applied for the validation studies of different road geometric design, with the aim of a low cost simulator. This paper describes the basic structure of the layers generated for developing scenarios and guidelines for the implementation thereof. The creation of the simulated scenario is very fast based on the geometric design software, and will make it easier to consulting firms to use the system for evaluating and auditing a particular route, obtaining reliable conclusions at minimal cost, even if the road is not actually built.

\section{METHODOLOGY}

\subsection{Simulation software requirements}

The programming environment developed in this research is based on Visual C ++2008 Express to run in real time. To generate meshes of objects in the environment, such as signs, walls, trees, etc. it will be used the 3D modeling Blender 2.70 and later. Python 3 it is used to process data and calculations offline. To support vehicle dynamic and their behavior with the collision system we use nVidia PhysX library 3.2. The audiovisual section rests with the Microsoft DirectX 8.1 libraries specifically for Direct3D graphics rendering and DirectSound for sound reproduction.

\subsection{Simulation scenario development}

To build virtual reality urban and interurban environments that already exist -or may be in the design phase-, are often used market edition and 3D modeling tools as 3DSmax or 
Blender. Alternatively it could be used gaming engines as Unity or UDK to simplify the design tasks. However, it is not possible to use these methods for environments of more than $100 \mathrm{~km}^{2}$, as the cost-time development and people involved is too high. Another problem that arises when using this type of modeling software is that usually suffers from technical limitations, such as excessive amount of memory required or limited precision floating-point numbers.

For this reason, in this research it has been employed another strategy to generate scenarios with low-cost familiar tools, and with minimum human supervision requirements, using as a basis the horizontal and vertical alignment of the road section, cartography and orthophotography of the area, inventory of road and environmental elements, etc. Given these data, it is possible to procedurally generate the geometry and texture of the ground. That is, you can automate the process by programming scripts to interpret the actual data and create entire virtual environment and resources needed for the simulation software.

Some solutions to design large scenarios consist on working with the viewing distance of details, so that nearby areas are perceived with a lot of detail, but with increasing distance the lower the resolution thereof. For this purpose a hierarchical subdivision of the space in a quadtree is performed to implement a system CLOD (Chunked Level Of Detail) (see figure 1a). Quadtree nodes are updated in real time, loading and unloading meshes, reducing detail depending on the distance to the vehicle. The union of all the meshes of different resolutions generates an anisotropic surface with higher density of polygons around the vehicle (figure 1b). The CLOD system methodology allows us to see areas several kilometers away maintaining a rate of frames per second controlled and acceptable meshes quality. When using lesser powerful graphics card, it can be reduced the workload by simply reducing the resolution index of meshes.
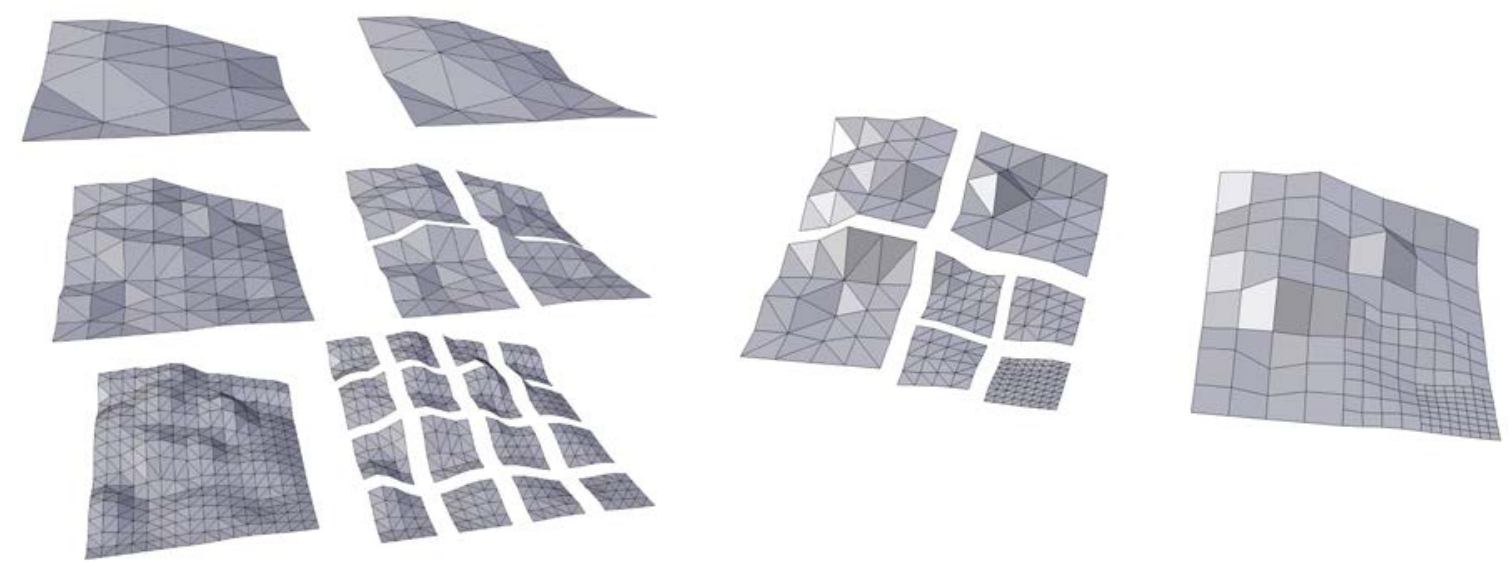

a)

b)

Fig. 1 - (a) Quadtree division for a chunked level of detail (CLOD) system; (b) CLOD system division applied to a terrain surface 


\subsubsection{Input Data Files}

To generate an interurban environment that meets our needs it will be necessary a map of heights to generate the field, information about the axis dimensions and camber of the road to be evaluated, an ortho-photo of the environment to generate terrain textures and positions of objects as signs, trees, safety fences, walls, etc. All these data can be extracted from various sources and with different formats. If the road network data already exist, these sources are usually published free of charge and frequently updated by public administrations. In case of the road network in which will be based the simulation scenario is under development, this information would be obtained and provided by market software for road geometry design, such as Civil3D or similar packages.

Figure 2 shows the structure and content of the multi-layer data package defined in our methodology need to create a new simulation scenario. To obtain the terrain geometry it is needed a height map in ASC format. This format consists of a header and a matrix. In the header are specified global map coordinates; the size of each cell in the array and the number of rows and columns are specified. From these data we can calculate the overall dimensions of the virtual environment. Each cell in the matrix gives us the height of the terrain, which can generate a huge mesh that accurately reproduced the topology. The similarity between the virtual and real ground depends on the cell size and altitude accuracy.

Road information is encoded in two files XLSX (figure 2). The first one stores the path of the center road axis, encoded in three fields: Station, $\mathrm{X}$ and $\mathrm{Y}$ coordinates. Dimensions are encoded in four fields of the second file: station, height on axis, left and right height. The camber is calculated from these three levels.
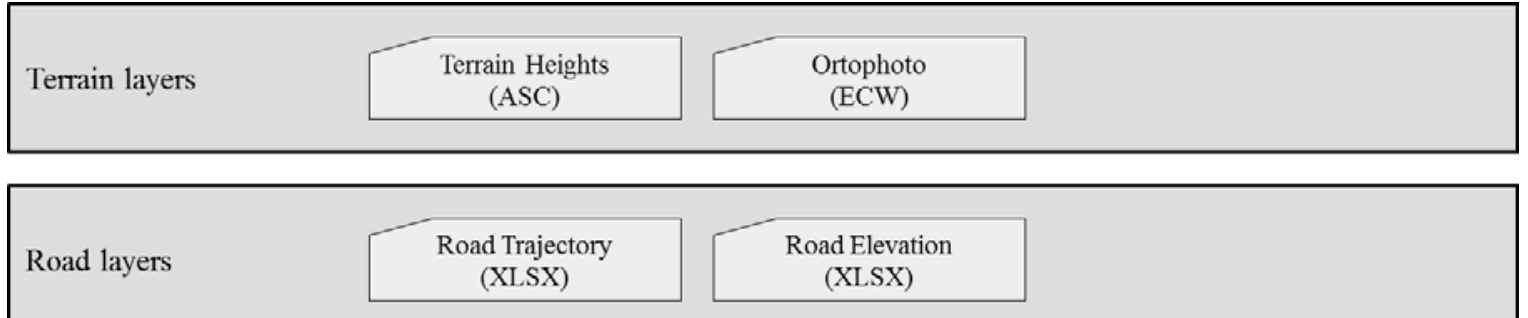

\begin{tabular}{|c|c|c|c|c|}
\hline Signaling layers & $\begin{array}{l}\text { Biondas } \\
\text { (DWG) }\end{array}$ & $\begin{array}{l}\text { Speed Sign } \\
\text { (DWG) }\end{array}$ & $\cdots$ & $\begin{array}{c}\text { Turn Sign } \\
\text { (DWG) }\end{array}$ \\
\hline
\end{tabular}

\begin{tabular}{|l|c|c|c|c|c|}
\hline $\begin{array}{c}\text { Tree Type 1 } \\
\text { (DWG) } \\
\text { (DWG) }\end{array}$ & $\cdots$ & $\begin{array}{c}\text { Bush Type 1 } \\
\text { (DWG) }\end{array}$ \\
\hline
\end{tabular}

Fig. 2 - Data processing layers 
Around the road all kinds of signs and vegetation are located. These objects, with relatively small size, have a shape, position and orientation to be specified when generating the complete environment of the scenario. To simplify the process and use familiar tools, in our case we used the AutoCAD DWG format program to separate data layers. Each object is encoded in a different layer using points, lines and polylines specifying their appearance.

\subsubsection{Input Files Processing}

The simulation software requires its own files and formats, so that is independent from actual file formats data environment. This is due to the original formats are not prepared to be treated together in real time, so we pre-process them to generate a more optimized and prepared files for CLOD system. These optimizations reduce the number and size of files, in order not to saturate the resources of the simulator computer and accelerate the process of loading and unloading in the background stage. The pre-processing is carried out in several stages, which are executed in code scripts. Each script takes some input files and transforms them into other files which, in turn, can be entered from other scripts. All this pre-processing is performed only once, taking several minutes to complete.

Figure 3 shows the inter-connection scheme between scripts and data files during the processing time. In the picture, gray rectangles represent scripts; everything else represents data files. Each script processes information as follows:

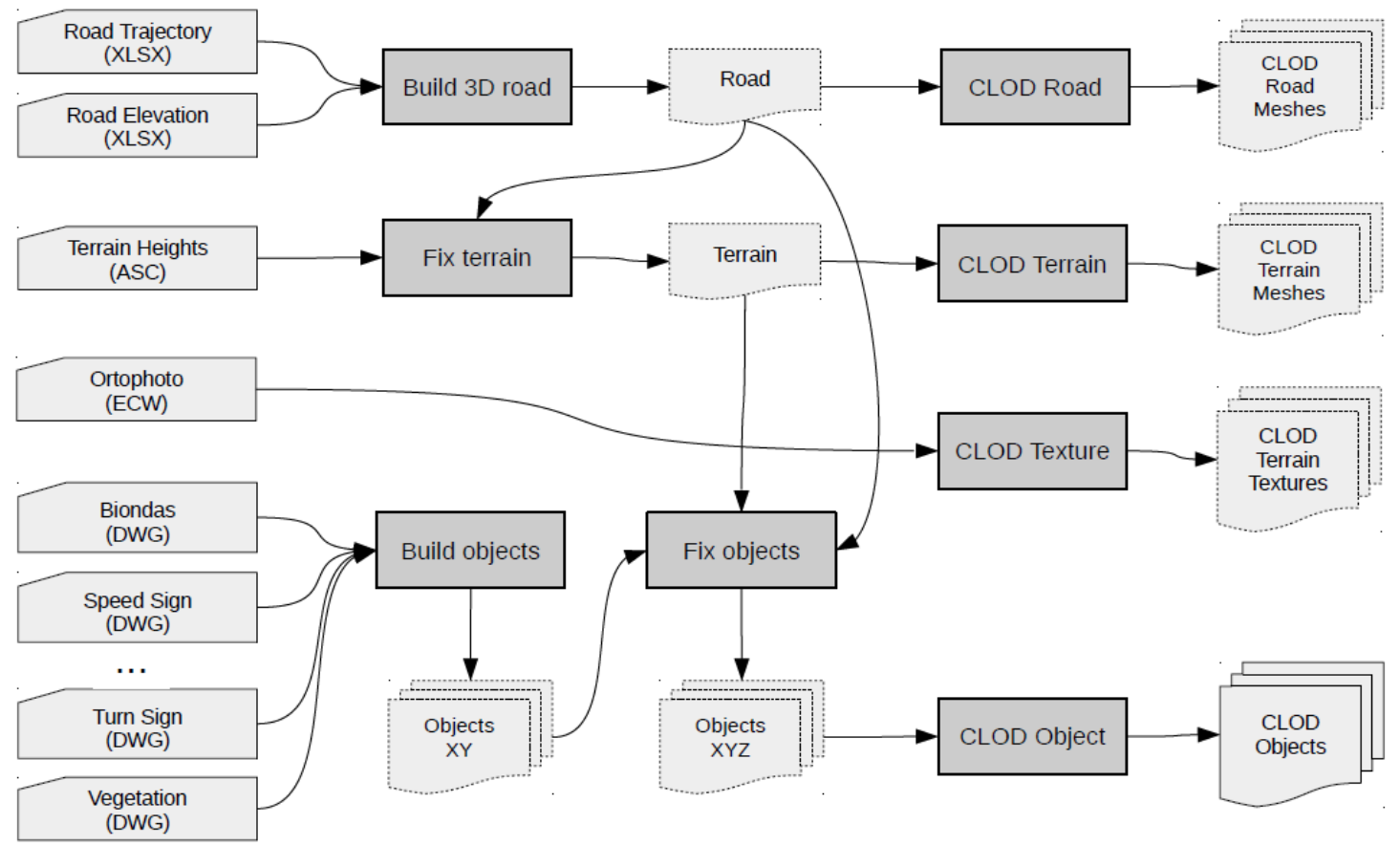

Fig. 3 - Data processing scripts

- Script "Build 3D road": It takes as input two files with information in format XLSX with road elevation and horizontal plane to generate a new combined file. At this point 
no mesh is generated, only information on the micro-sections of the road is stored to simplify the subdivision into cells and generate meshes in next phases.

- Script "Fix terrain": Responsible for superimposing the road over the ground in order to correct the vertices of the heights map that rise above the road. This problem arises because the road has more resolution than the terrain or because data can come from different sources, giving similar measurements but not necessarily accurate. Therefore it requires as input files the terrain heights and the temporary file with the microsections of the road. As output we get a new heights mapping file that replaces the original in the following phases.

- Script "Build objects": decodes all information layers from AutoCAD DWG files to export the points and polylines of objects in the environment. The result is a new temporary file with the position of each object on the XY plane.

- Script "Fix objects": This phase extends the previous to provide orientation and objects position. The new position is recalculated based on the map of terrain height and distance from the axis of the road; for example, signals are repositioned to the sides of the road. The orientation of some objects is randomly distributed, such as trees, safety fences are oriented perpendicularly to the axis of the road, and other objects should be oriented in the opposite direction to the line in which is circulating the driver, as the signals curve. The new file generated contains the same information as above plus the XYZ position and orientation.

- Script "clod terrain": is responsible for dividing the entire ground surface in a mesh with progressive levels of detail to be used in the CLOD system. As input files it is only needed a height map to indicate the minimum mesh size.

- Script "clod road": The purpose is the same as the previous script, but in this case micro-sections of road are used. Once generated all levels of detail by combining micro-sections, we proceed to generate meshes. It takes as input the temporary file of road settings to indicate the minimum mesh size and a output folder to store the generated meshes.

- Script "clod texture": As input files requires the ortho-photo in ECW, configuration parameters to indicate the offset relative to the ground and an output folder format.

- Script "clod object": Since objects are small in size compared with the terrain and the road, it is not necessary to subdivide their mesh to use the CLOD system. However, it is necessary to group them by zones and load only the areas around the vehicle.

All these scripts must be later "cooked" to optimize their computing treatment. So, in order to send the final processed orders to the final simulation scenario, it must be done another internal step to improve the meshes created. Figure 4 shows the relationship between the multi-layered processed output files with the simulator software control. The scripts employed in this case are the following:

- Script "Cooking": it refers to the treatment performed by PhysX physical library with the aim of simplifying and generate meshes collision, calculating convex volumes and 
adding metadata to accelerate collision calculations. The result is a proprietary binary library file.

- Script "Compressing": The original size of the ortho-photo in ECW format is about 2.4GB. After subdividing and generate all the detail levels it could be reached 5GB. It is highly recommended to compress terrain textures to JPG to reduce the required total capacity hard disk for a faster loading data in the simulation software.

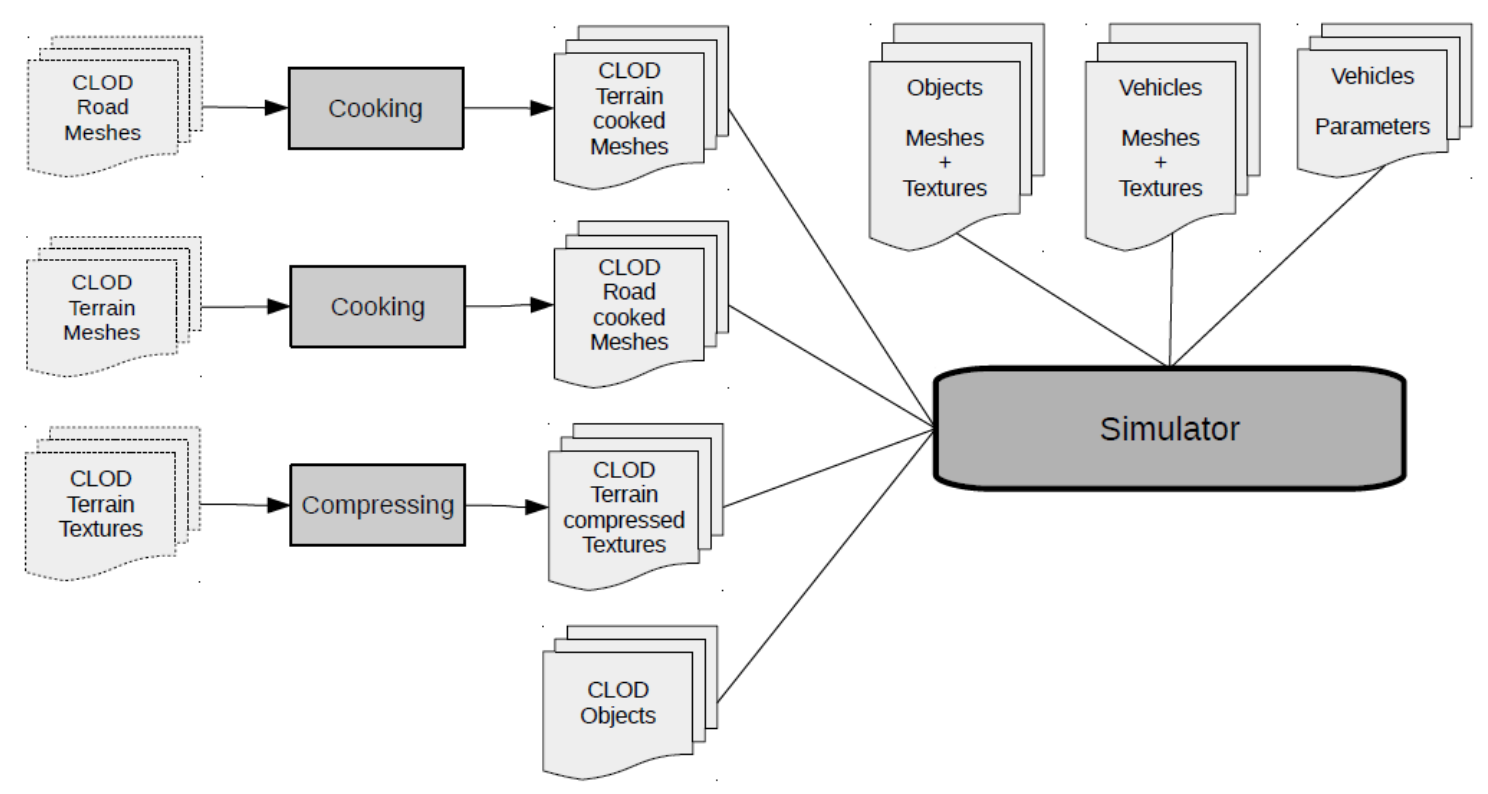

Fig. 4 - Data processing interacting with the simulator

\subsubsection{Other Input Data Files}

The vehicles are configured in a document with XLSX format, taking several books to parameterize the various parts and vehicle components. The physical library supports all of these parameters, so that it can be set infinite number of vehicle models. Along with the geometric description of the body, the physics engine can determine the physical and dynamic vehicles behaviour in the simulation. To model the vehicle chassis and convex collision volumes it could be used Blender software. This program can also indicate the wheels position and the centre of gravity of the vehicle. In addition to the rendering system, it will also need a graphical model of the vehicle.

\subsection{Road Geometry Data to validate the scenario design}

The road section selected for the validating process of the simulator scenario developed was the road CV-35, from Losa del Obispo (station 53+500) to Titaguas (station 83+700), located in the Valencian Community (Spain). Lane width is $3.25 \mathrm{~m}$, while shoulder width is $0.25 \mathrm{~m}$. The ADDT for this road is 2012 vehicles per day. This road was chosen due to section it has three segments with different geometric characteristics which cover a wide range of curves. The first one extends from Losa del Obispo to Chelva - basically composed of isolated high radii curves and low grades-, a second segment located between 
Chelva and Tuéjar, characterized by successive low radii curves with short length and moderate grades, and finally a third segment which begins in Tuéjar and ends in Titaguas, that includes low radii curves, high sinuosity and, above all, large grades. The total length is $30.185 \mathrm{~km}$, but the effective length is $28.877 \mathrm{~km}$ because there is $1.308 \mathrm{~km}$ of urban road in the road section.

In our validation study cartography and ortho-photography were downloaded free from the website of the National Plan of Air Ortho-photography (PNOA). The cartographic files were obtained based on a LIDAR with a density of $5 \mathrm{~m}$ mesh size, which is proper for this project. The horizontal alignment was obtained according to the methodology proposed by Camacho-Torregrosa et al. (2013). In addition, the vertical alignment was extracted from GPS data of the tests. The setting of the vertical alignment was carried out with the same program used to the setting of the horizontal alignment, but this module of the program was designed specifically for this research. With both alignments, the road section study was restituted in AutoCAD Civil 3D.

On the other hand, a CAD file was created in order to collect the different road and environmental elements, such as trees, traffic signs (limited speed, recommended speed, dangerous curve...) or safety barriers.

\subsection{Driving simulator tool}

The experimental tool used for validating the scenario designed was the $\mathrm{SE}^{2} \mathrm{RCO}$ driving simulator, which consist on an interactive fixed-base driving simulator (see figure 5). It is composed by a simulation computer, which provides the graphics performance required for the implementation of the simulation software; data collection in real time; wireless router; three-screen-display monitors $1.80 \times 0.34 \mathrm{~m}$ with $120^{\circ}$ of the field of view; Matrox TripleHead2Go graphics card; sound stereo system; steering wheel, pedals and gear shift of a Citroen Saxo and generic adjustable seat. All this provides a view of the road and the environment very close to the real conditions. The equipment of the simulator allows collect many variables, such as speed, location, azimuth or lateral speed, with a frequency of $10 \mathrm{~Hz}$.

In addition, the simulator has been sensorised by load cell to measure forces at brake pedal; potentiometers for measuring displacement in the three pedals; micro-switch for detected the gear-box lever position; encoder for measuring the steering wheel angle; and torque sensor for measuring torques on the steering wheel.

\subsection{Validation experiment scheduled}

\subsubsection{Data Collection}

The main variable to consider for the validation of the $\mathrm{SE}^{2} \mathrm{RCO}$ driving simulator is the speed. The methodology to apply in this study was presented by García et al. (2015). The data collection was carried out by three VIRB Elite cameras, which allow for obtaining 
continuous speed profiles. These cameras were placed in the car of each participant. The experiment was scheduled to compare between the continuous speed profiles obtained in the field study and in a simulator. In this regard, the average and operating speed on 79 curves with a radius from 40 to $520 \mathrm{~m}$ and 52 tangents with lengths ranging from 120 to $1500 \mathrm{~m}$ was looked for. The test consisted in driving forward and backward along the entire road segment. Before performing the test, each driver was informed about the aim of the research and what had to do. Then, the driver had to sign an agreement.

This study was performed by 28 drivers for 7 days between March and April 2014 under favourable weather conditions and daylight. In addition to the data collected by the cameras, information about the driving experience, road familiarity, dizziness and workload was also collected through a survey. The goal of this survey was to characterize the sample of drivers based on different parameters and check drivers' consistency and the naturalness of the test. All drivers answered that they performed in a natural (or quasinatural) way, with a low workload increase due to the experiment. In other case, the nonnatural driver would have been removed from the analysis.

This testing process was repeated in the simulation scenario developed in the project that reproduced the same places an itinerary. The methodology applied for developing the scenario was the presented in this paper. Finally, the virtual reality was designed without opposing traffic, under favourable weather conditions and dry pavement.
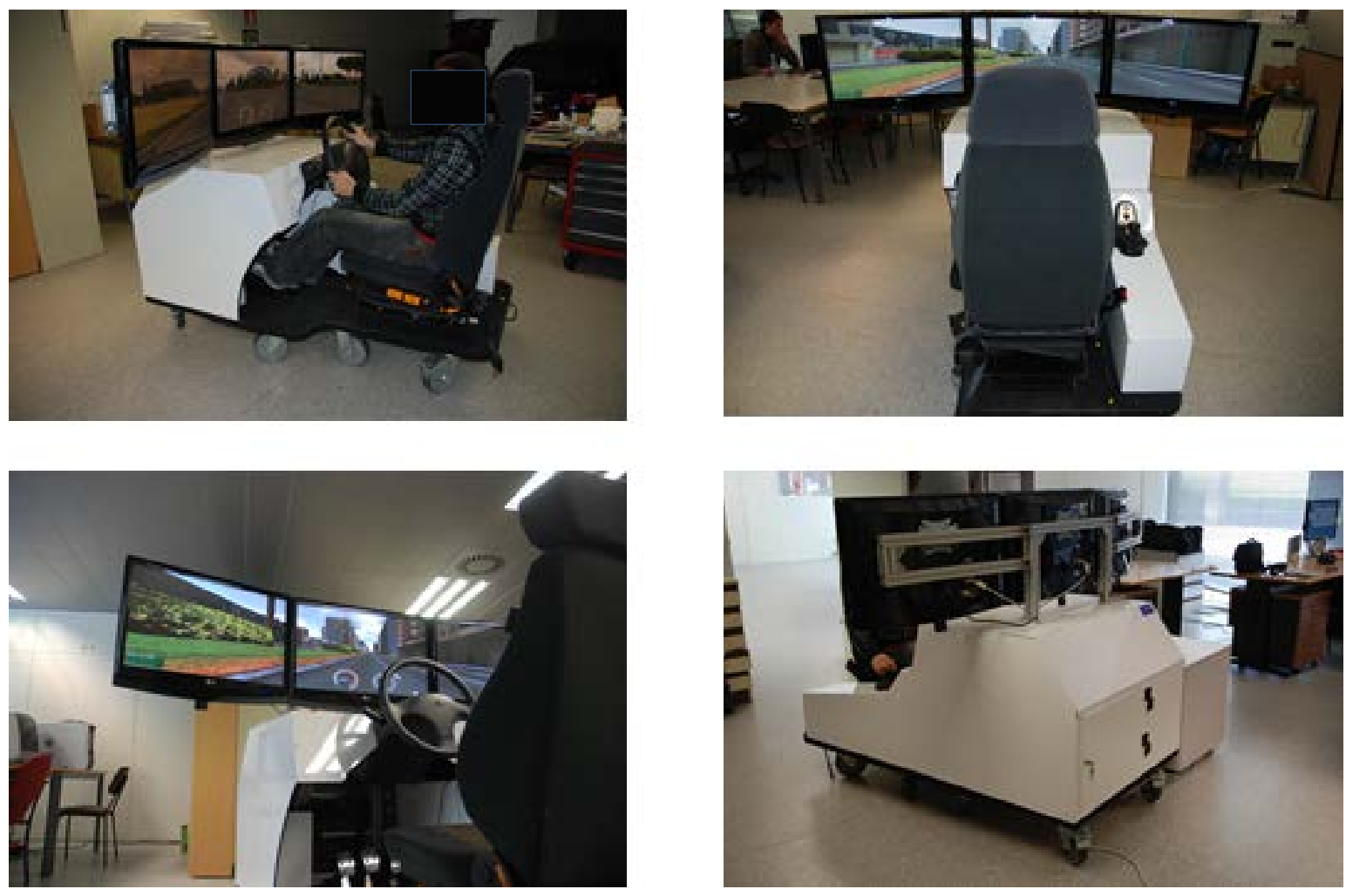

Fig. 5 - SE ${ }^{2}$ RCO driving simulator 


\section{RESULTS}

The results obtained in this experiment were presented by Llopis-Castelló et al. (2016). This analysis showed that an absolute validity was obtained in 118 configurations according to the average speed. In these alignments, when the speed in the simulator was lower than $87.3 \mathrm{~km} / \mathrm{h}$, the average speeds in the driving simulator and in the real road were similar. When the simulated speed was greater than $87.3 \mathrm{~km} / \mathrm{h}$, usually on long tangents, this speed was greater than the real speed. So, as the alignment configuration becomes less difficult, the difference between the speed observed in the driving simulator and the speed developed in the field study is greater because the perceived risk in the simulator is lower than in the real world.

From a relative validity perspective the comparison between the operating speeds showed that the $\mathrm{V}_{85 \mathrm{r}}$ was $5.24 \mathrm{~km} / \mathrm{h}$ lower than $\mathrm{V}_{85 \mathrm{~s}}$, what demonstrated that the $\mathrm{SE}^{2} \mathrm{RCO}$ driving simulator is a useful tool for driving speed behavior research, since there is a high correlation between the driver behavior in the simulator and the real road.

From the driving simulation scenario perspective, the graphics result of the multi-layer methodology applied can be observed in figure 6 . This scenario was designed to reproduce the same road of the field test, approximately $30 \mathrm{~km}$ in length along CV-35 from "Losa del Obispo" to "Titaguas" cities (Valencia, Spain). The level of detail in the plan view of some sections has been captured in Figures 6a and 6b. The integration of different objects in successive layers that have shaped the virtual scenario - safety fences, defenses, signaling, road markings, vegetation, etc.- are showed in figures 6c, 6d and 6e.

The degree of realism developed for the virtual scenario was defined based on surveys of all participants in the experiment. These surveys sought to determine the symptoms of adaptation to the simulation and perception of the simulated reality -naturalness of participants in driving, workload, familiarity with the road, environment etc.- made after their participation in driving simulator test.

The results showed that $62.5 \%$ of drivers assessed the quality of the virtual environment created as middle, while $33.3 \%$ considered high. Approximately $80 \%$ of participants felt that the degree of similarity between the real driving task and the driving simulator could be considered as middle or high. Regarding the assessment of the workload and the ease of driving, most participants considered average; these being very close to those obtained in the field study results.

\section{DISCUSSION}

The possibility of working with software packages, that theoretically enables the exchange of data formats from different databases for simulation environments design, has been analysed in the past years. Some cases as RoadXML format developed by Oktal, or OpenDrive from VIRES, have demonstrated that a new methodology for developing road 
simulation scenarios based on the use of multiple layers-file format system is a fairly good procedure to do so. In these cases, data often come from different sources but known formats. Nevertheless, the participation and collaboration of private intermediate agents that manages and control access to simulation scenario file sharing, could be seen as a barrier and a drawback for real interoperability.

The same multi-layer methodology for developing virtual driving simulation scenarios has been used in this research program by the IDF and GIIC teams from UPV. But in our case, we have used common formats (XLSX, DWG, etc.) that can be edited using different programming libraries and software packages, both free and owners.

By using scripts to treat these formats, we can quickly adapt them to other formats, opening the door to different free data sources. This allows us to generate scenarios of different areas -urban, suburban, rural, highway, etc. - in an automated procedure. After collecting the necessary data and run the scripts, we get a scenario prepared to be executed by the simulation software. Since the data obtained came from updated measurements, the scenario obtained is quite faithful to virtual reality representation.

With this methodology the user can interact freely and easily with the virtual simulation cab, and visualize the road and surroundings several kilometers away thanks to CLOD system implemented. The physics engine integrated in the simulation software allows the vehicle to dynamically behave realistically, taking into consideration the unevenness, camber and frictional forces on the wheels.

Relative validity of the simulator tool has been achieved according to the operating speed and the average speed, since the speed determined in the simulator presents a high correlation with the speed identified in the field test. Further, the statistical analysis showed the absolute validity of the simulator regarding the average speeds, except in those configurations that were located near important intersections of the real road, where the simulated speed was very greater than the real speed. The quality of the virtual environment developed and the similarity of driving task between the simulator and the real world has help in the research done to fulfill the objectives pursued.

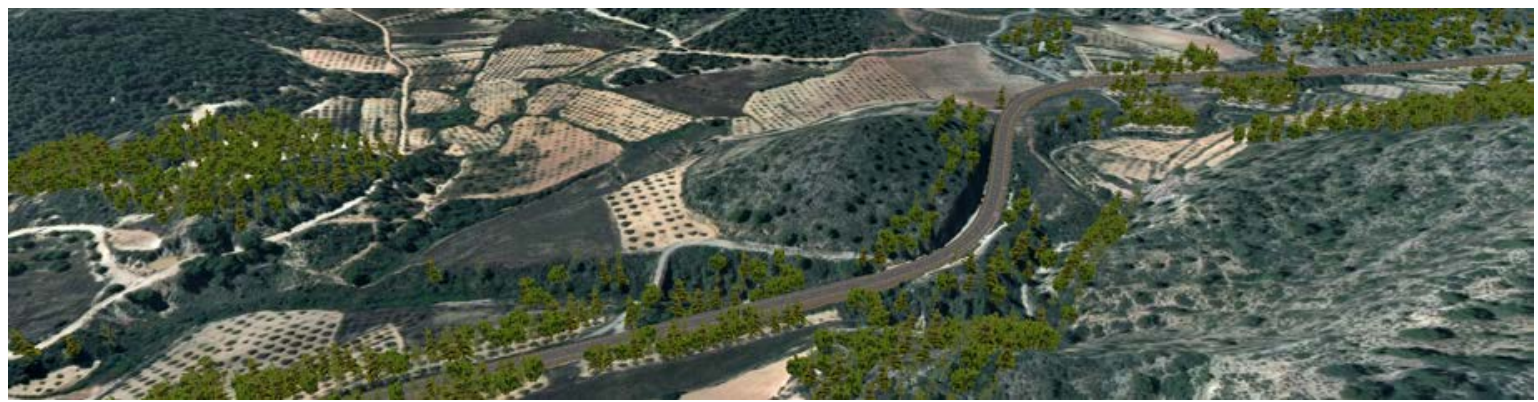

a) Aerial view of a section of the road. The road and terrain are overlapped with fair realism despite the data come from different sources. 


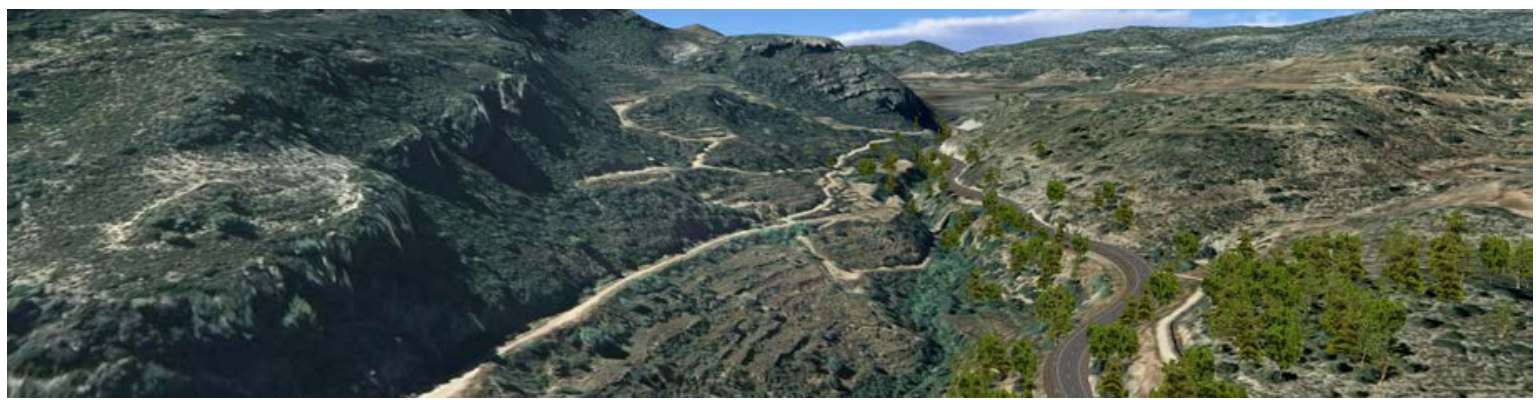

b) Aerial view of a large area. The CLOD system allows near and far scenario view (many $\mathrm{Km}$ ) if saturate the hardware simulator.

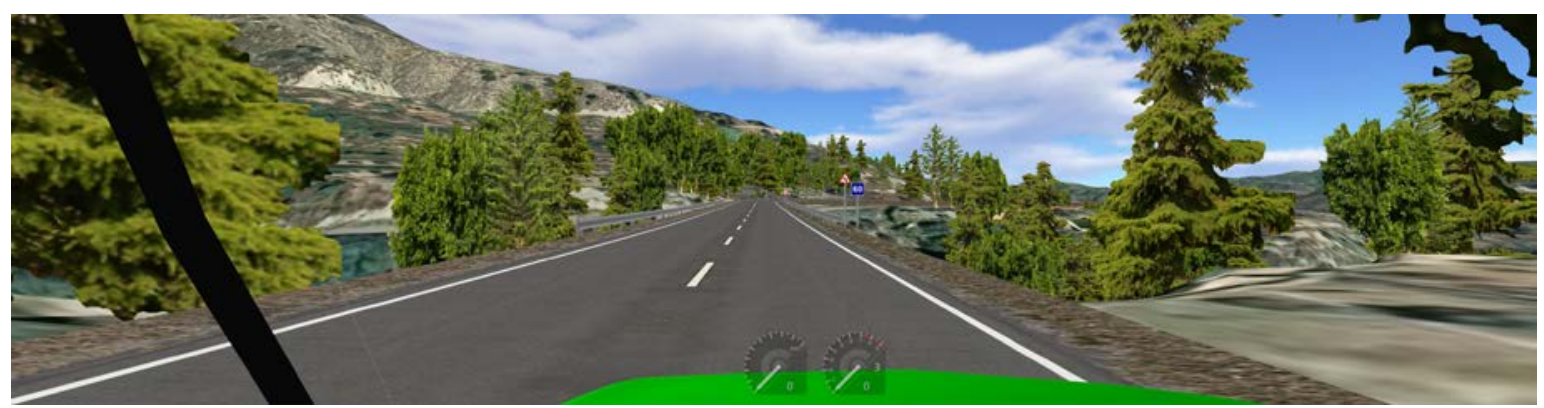

c) Driver's view of a straight section of the road. The vegetation adapts to the terrain and the environment.

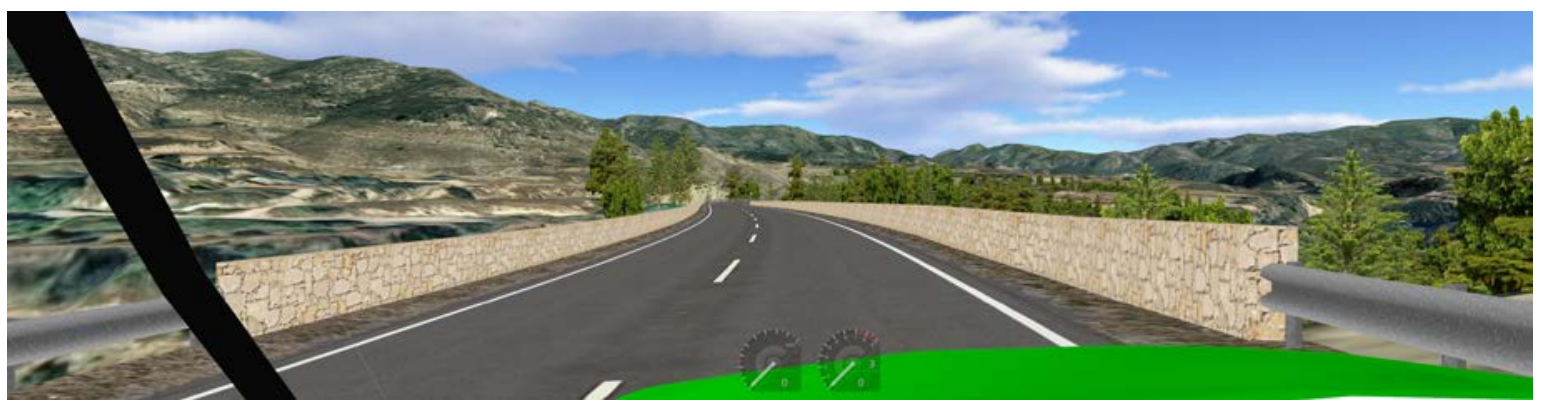

d) Driver's view of a high safety section with walls alienated to the axis of the road.

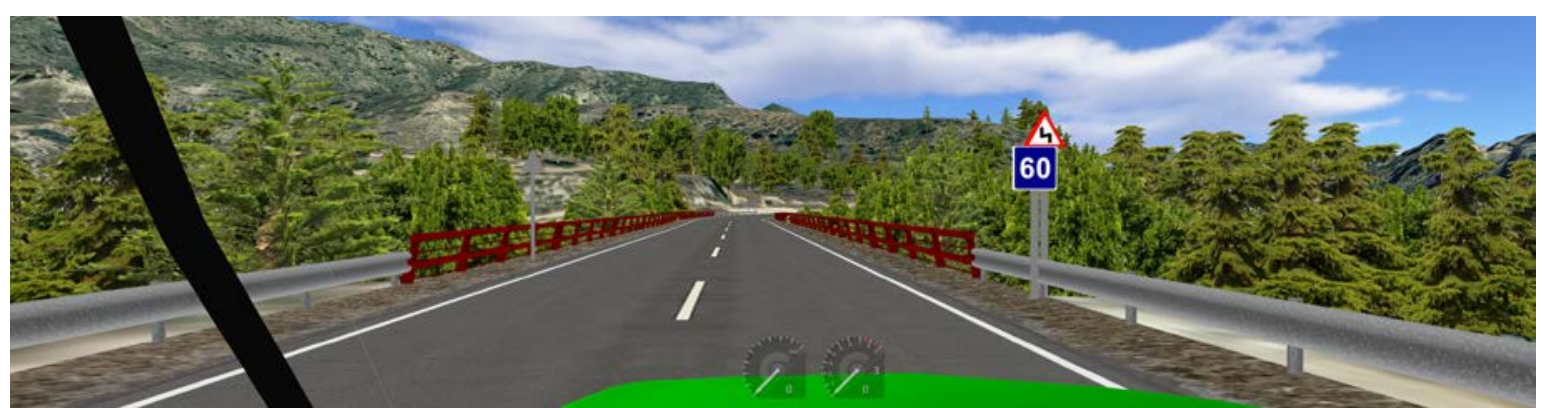

e) Driver's view of a large section with guardrails oriented in flow direction signals.

\section{Fig. 6 - Images from the simulation scenario developed}

\section{CONCLUSION}

This new methodology for developing scenarios has been applied by the Institute of Design and Manufacturing (IDF) of the Polytechnic University of Valencia (UPV), in collaboration with the Engineering Research Group Highway (GIIC) of the UPV, for the 
validation studies of different road geometric design, with the aim of a low cost simulator. This paper has described the development of a real simulated scenario. The procedure used is very fast based on the geometric design software, and will make it easier to consulting firms to use the system for evaluating and auditing a particular route, obtaining reliable conclusions at minimal cost, even if the road is not actually built.

Drivers' perception supported the validity derived from speed analysis, so the most of volunteers assessed the quality of the virtual environment and the similarity of driving task between the simulator and the real world as medium or high. Only drivers who suffered dizziness evaluated the simulator features negatively.

\section{ACKNOWLEDGEMENTS}

Authors would like to thank Polytechnic University of Valencia (UPV) that subsidizes the research project CONSIM (PAID 05-2012).

\section{REFERENCES}

ALLEN W. et al. (2000). A Novice Driver Training Experiment Using Low Cost PC Simulation Technology. DSC2000 International conference, Paris, 6-8.09.

BHATTI, G.; JESSEL J.P.; BREMOND, R.; MILLET, G.; VIENNE, F. (2012). Towards the development of a user interface to model scenarios on driving simulators. Driving Simulation Conference, 6-7 Sept. 2012. Paris, France.

CAMACHO-TORREGROSA, F.J., PÉREZ-ZURIAGA, A.M.; CAMPOY-UNGRÍA, J.M.; GARCÍA-GARCÍA, A. (2013) New geometric design consistency model based on operating speed profiles for road safety evaluation. Accident Analysis and Prevention, Vol. 61, pp. 33-42.

CHAPLIER, J.; THAT, T.N.; HEWATT, M.; GALLEE, G. (2010). Toward a standard: RoadXML, the road network database format. Proceedings of the Driving Simulation Conference Europe. Paris, France.

DUPUIS, M.; STROBL, M.; GREZLIKOWSKI, H. (2010). OpenDRIVE 2010 and Beyond - Status and future of the de facto Standard for the description of road networks. Proceedings of the Driving Simulation Conference Europe. Paris, France.

GARCÍA-GARCÍA, A.; CAMACHO-TORREGROSA F.J.; MARÍN-MORALES, J. (2015). Experimental determination of the inertial operating speed for consistency and segmentation analysis. Transportation Research Board 94th Annual Meeting. No. 15-4079. HIBLOT, N.; GRUYER, D.; BARREIRO, J.S.; MONNIER, B. (2010). Pro-SiVIC and Roads, a software suite for sensors simulation and virtual prototyping of ADAS. Proceedings of the Driving Simulation Conference Europe. Paris, France.

LLOPIS-CASTELLÓ, D.; CAMACHO-TORREGROSA, F.J.; MARIN-MORALES, J.; PEREZ-ZURIAGA, A.M.; GARCIA, A.; DOLS, J.F. (2016). Validation of a low-cost driving simulator based on continuous speed profiles. Proceedings of the TRB Conference. TRB Paper 16-1441. USA.

NORDMARK, S. (1990). The VTI Driving Simulator - trends and Experiences. 
Proceedings of Road Safety and Traffic Environment in Europe. Gothenburg. Sweden.

REED, M.P., GREEN, P. A. (1999). Comparison of driving performance on-road and in a low-cost simulator using a concurrent telephone dialling task, Ergonomics, 42(8) 10151037

SCHINDLER, J.; HESSE, T. (2014). Coping with complex driving scenarios: exploratory scenario design. Driving Simulation Conference, 4-5 Sept. 2014. Paper no 8. Paris, France. VERWEY, W.B. (1995). The Control of Input Devices by people driving with and without adaptations. DRIVE II PROJECT V2032 TELAID Deliverable No. 9. Validation of ATT Systems for Drivers with Special Needs through Driving Simulator Tests. Brussels.

WEIR, D.H.; CLARK, A.J. (1995). A Survey of Mid-Level Driving Simulators. SAE. SP1080. Vehicle Computer Applications. Vehicle Systems and Driving Simulation. Warrendale, USA. pp. 53-73 